\title{
LA PÁGINA dE MALLARMÉ O EL SIGNO MATERIAL
}

\author{
Jesús Camarero \\ (Universidad del País Vasco)
}

A Jan Baetens

En el mes de mayo de 1897 la revista Cosmopolis publicaba el poema «Un coup de Dés jamais n'abolira le Hasard» de Stéphane Mallarmé. En esta composición se ponen en práctica unos principios teóricos sobre el signo gráfico (tipografía incluida), sobre el volumen-libro (paginación) y otros componentes bibliológicos que resultan de absoluta novedad en el ámbito literario; donde precisamente siempre se utilizó la escritura y su inscripción en la página como método de elaboración de la obra, pero donde no se había hecho hasta ahora ningún intento para decir y mostrar eso mismo que se hacía y escribir utilizando los recursos disponibles, salvo quizá algunos casos aislados (y no tan intensos) como el de Laurence Sterne en su Tristram Shandy, en el siglo XVIII. Es decir que hasta aquella fecha la consideración del signo como entidad lingüística y escritural, como fenómeno de representación que además se escribe y representa al mismo tiempo, había sido prácticamente nula y lo que hoy llamamos «signo» (o mejor, la reflexión sobre aquello que produce significado o que es comprensible en el ámbito de los sistemas de significación) no era más que una nebulosa vacua sobre la que nadie se había detenido demasiado, bien por no considerar excesivamente la dimensión formal de lo escrito, bien porque se prestaba más atención al contenido, a lo representado por el signo (la lengua y lo evocado a su vez por ella, por ejemplo) y no ya tanto al signo mismo y su entidad específica. En este sentido, la obra de Mallarmé pre- 
senta una factura, una forma, una configuración textual y escritural que no puede en principio dejar sin reflexión al lector y que, más allá de la lectura superficial o apresurada del lector no advertido o descuidado, obligaría a una reconversión de ideas básicas referidas al concepto de literatura, es decir, a una teorización inexcusable sobre el texto, la escritura, el acto de escribir y producir significado. Y más concretamente sobre una especificidad material del elemento sígnico, sobre una especie de referencialidad no buscada en lo externo al signo (que siempre es otra cosa que no es él), sino en su propia esencia, es decir, una actualización conceptual de todas las formas, funciones y performancias que posee o que genera la entidad llamada signo. Porque, además, cuando de la escritura se trata, no se puede obviar fácilmente la cuestión de la inscripción del signo sobre un soporte, sobre algo que es palpablemente material, no se puede dejar de lado el fenómeno de materialización de una entidad abstracta que necesita cristalizar y conformarse perdurablemente. Al descender y depositarse sobre el soporte, ese trazo de la escritura que es la letra y el componente gráfico, adquiere entonces un aspecto o dimensión material que antes no tenía, de modo que podemos hablar ya de un objeto tangible e incluso transformable. A partir de aquí entramos en una consideración de lo literario que no es definible solamente por una relación del tipo escritura/lectura, sino que además debe completarse por lo menos con otras consideraciones (también de orden semiótico): la inscripción, la visualización, la materialidad, la legibilidad, el objeto-volumen (libro), etc. El texto, producto final de la escritura inscrita en/sobre el soporte-página, adquiere entonces una dimensión que va más allá de aquel mensaje representado y tiene como implicación más próxima el hecho de poder considerar el texto como un objeto material, cuyo funcionamiento no sería muy diferente al de otras producciones pictóricas o escultóricas, es decir, dotado ya de una plasticidad que antes no le caracterizaba precisamente. Esta plasticidad del signo escritural y literario no puede tener, sin embargo, el mismo grado que en las artes consideradas plásticas desde siempre, porque la materialidad del signo carece de la motilidad del óleo, le falta la ductilidad del bronce fundido, no se deforma, ni permite una modelación a mano; pero es plástico, porque adquiere formas muy diversas (el tipo y paso de letra, densidad, enfatización, giro sobre el eje de la página), puede tener apariencias variadas (tintas, páginas en negro, sobreimpresiones con otros signos), se combina a veces con elementos pictóricos (líneas, trazos) y se deposita sobre un soporte cuya textura, densidad o color no tienen prácticamente límites. En este sentido, el Diseño Gráfico por ordenador está demostrando que es posible la manipulación de cualquier forma hasta límites inimaginables y el signo escrito no es una excepción, sino más bien el objeto primero y más importante de este nuevo sistema de creación por medios informáticos, de modo que se puede afirmar que el grado de manipulación de la «materialidad» de ese signo puede alcanzar los niveles de las otras artes plásticas, sin envidiarles además los logros conseguidos gracias al software. 
«Un coup de Dés...» es un texto configurado de acuerdo con unas pautas especificas de inscripción en el soporte (las páginas del volumen, del libro). Una vez abierto el libro, la página izquierda queda vacía para dejar que el título se coloque en la página derecha, pero sólo parte del título y nada más que ese fragmento, porque el texto realmente empieza al pasar la hoja y ver las dos páginas siguientes simultáneamente. Este primer plano sobre el que se realiza la visualización está constituido entonces por dos páginas numeradas de modo distinto y cosidas (o pegadas) en el lomo del volumen. En la edición de las obras completas de Gallimard/Pléiade (así como en otras ediciones y traducciones) el texto se organiza siempre del mismo modo y el trabajo del maquetista deberá respetar una serie de pautas muy precisas a la hora de componer las galeradas. A lo largo de veinte páginas (es la extensión ideal de la obra para el autor), desde la página 458 hasta la 477 (ed. Pléiade), las páginas se organizan por parejas en cada nuevo paso de hoja, de modo que obtenemos diez dobles páginas que Mallarmé utiliza también como unidad de composición.

\section{LA ORGANIZACIÓN DEL TEXTO}

La organización interna del texto se realiza en base a dos componentes. El primero de ellos, la página, no deja de ser el habitual, pero su tratamiento específico demostrará capacidades ignoradas o no utilizadas hasta la fecha de 1897. El segundo es el clinamen junto con sus distintas variantes, efecto plástico y dinamizador del texto y de la página, artificio (común en algunos textos oulipianos de G. Perec) que consiste en utilizar la misma escritura para representar además la trayectoria de lo escrito y, básicamente, la «inclinación» o deriva que resulta del fenómeno escritural occidental al disponer los signos de izquierda a derecha y de arriba a abajo. La organización del material escritural (las palabras, los versos, más como unidades de consumo visual que como estructuras sintácticas) no es típica, no pretende construir estrofas ni otro tipo de esquema preexistente, al contrario, desea poner en evidencia que se trata de un material "a disposición», que es utilizable, que de hecho lo está manipulando, que hay un modo de decir poesía por medio de una "sintaxis» nueva y diferente.

La página ha acompañado y sigue acompañando todo acto de escritura. Todo signo escrito es permanente gracias al soporte que lo contiene de algún modo y ello puede definir un tipo de relación entre el mismo signo y el soporte sobre el que se inscribe. De tal manera que Mallarmé manipuló o alteró esa relación cualitativamente, obteniendo un juego lectural basado en las posibilidades de disposición y combinación que ofrece siempre el trabajar con un volumen (libro). En la página III, «LE MAITRE» no busca seguir su frase por debajo, en su misma página, sino que tiende espontá- 
neamente (cálculo y provocación, no azar) a continuarla en la parte derecha, en una página distinta (463 y no ya 464); además, encuentra enfrente no uno sino dos versos con los que puede combinarse perfectamente, eligiendo el primero o el segundo de ellos sin que por ello se altere otra cosa que la sublime capacidad de generar sentido a raudales en un efecto de espaciamiento o dispersión de la lectura, que ya no utiliza la linealidad consecutiva para producir significado, sino que juega a deslizarse por el soporte buscando nuevas posibilidades de asociación de significantes. Entramos pues en una sobredimensión combinatoria de esta disposición del plano lectural que contiene dos páginas en vez de una; en todo el texto constituido y contenido en estas dos páginas de la página III, se producen multitud de posibles combinaciones: "LE MAITRE» tiene dos versos enfrente a la derecha, "surgi...» dos o tres a la derecha, «que se...» encuentra el verbo también a la derecha, «comme on...» tres versos a su derecha, «l'unique...» tiene enfrente «être...», seguido de «Esprit» y tres líneas más, para volver luego a la izquierda con «hésite», el verso debajo de él y luego a la derecha, volviendo de nuevo la izquierda con "plutôt» y el clinamen de su misma página 463. Contrariamente a lo que ocurría en la página anterior (en la que se apologizaba del clinamen y de la transgresión de la página-soporte), en la página IV hay una búsqueda de la estructura horizontal de la escritura $y$, en cierto modo, una recuperación del verso convencional, aunque se reconoce en todo momento la factura o modo de hacer mallarmeana; al final de la página 464 (la de la izquierda del plano lectural) «folie» lanza una proyección o conexión hacia la otra página, en la que encontrará el verbo "N'ABOLIRA», pero no el comienzo de esa otra página (es decir, arriba) sino justo al final de ella y transgrediendo igualmente la disposición del signo en el soporte y el orden interno que organiza la lectura normal. El mismo autor, en su prefacio al poema adelantaba ya que no se trataba de «traits sonores réguliers ou vers» - como pueda ocurrir en la poesía convencional - sino que se trata según él de «subdivisions prismatiques de l'idée» (1945a: 455). En la página VI, «sauf», junto al hemistiquio de la costura de ambas páginas - con todas las precisiones de que da cuenta Papp (1989: 193-206) respecto al fenómeno bibliológico-, es el anuncio de una cita sin puntos suspensivos, ni dos puntos, ni comienzo de comillas en el otro lado; es simplemente decir que se dirá el texto de ahí al lado (por desplazamiento en el espacio del soporte, dentro del volumen), saltando por encima de la costura (que aquí casi ejerce funciones de signo tipográfico). Un trazo puro que viene a unirse en un tándem tópico con el otro texto, dando lugar a la locución adverbial con valor conjuntivo «sauf que», que se lee de un tirón como si nada existiera en medio. Así se produce la fusión de dos miradas del volumen en una sola o lo que es lo mismo, la desaparición de un modo de organización interna del volumen y su sustitución por un único plano de lectura, en el que lo que cuenta es ese plano conformado por dos páginas desplegadas (el volumen abierto, la obra "abierta») y cuya costura se hace invisible, aunque tanto el escritor como el lector sean conscientes de su existencia y ello llegue a contar en algunos 
momentos de la creación y su consumo. En la misma página, «plume solitaire éperdue», en cursiva, prácticamente aislada como frase en medio del blanco vacuo de la página izquierda y casi contradicha por ese "sauf», al límite del hemistiquio que permitirá el acceso al otro lado («sauf» está más ligado al texto de la derecha que a la frase que intenta delimitar), no es una entidad lingüística común, no constituye un significado representativo de una idea, es puro signo, es una pluma peligrosamente aislada en medio del soporte; sólo el «sauf» marginal y huidizo podrá proporcionar una posibilidad de ayuda. Así se constituye el acto meta-representacional de esta escritura, del verdadero «coup de dés» que es la apuesta por la obra, sobre todo cuando las circunstancias son tan adversas y cuando se es consciente de que la limitación es nada más y nada menos que el Todo, el azar infinito. En la página IX, la frase: «RIEN N'AURA EU LIEU QUE LE LIEU», tendida de un extremo al otro del plano lectural, está estructurando todo el texto al mismo tiempo que organiza los diferentes trayectos del ojo y de la lectura, fijando un posible precedente del poema «Pectoral primero» de J. Cortázar (1985: 269-271): en este poema «en árbol», que ocupa también dos páginas del mismo plano visual y lectural, dos frases, «en su lecho de arena se adormece / una mujer desnuda en una playa», son el punto de partida de otras frases o versos que surgen de ellas mediante la trayectoria marcada por unas flechas. De este modo, el texto matriz de los dos versos iniciales de Cortázar así como la frase de Mallarmé citada un poco más arriba vienen a convertirse en elementos generadores y organizadores de un entorno textual más complejo, logrando estructurar además la significación mediante recorridos debidamente calculados a partir de los datos del soporte. En la misma página, la repetición de «LIEU» deja insistir sobre la idea misma del espacio sobre el que se produce la inscripción, el soporte permite la alusión que se hace a su uso mediante la ubicación del otro espacio de los signos. La estrategia de la frase-verso tendida de un lado a otro se vuelve a repetir en la página $X$, con «EXCEPTÉ PEUT-ETRE UNE CONSTELLATION», combinado con un clinamen suave en la parte izquierda que va deslizándose hacia la derecha, como buscando el cierre del poema en la frase final, del mismo modo que el título diseminado (véase más adelante) recorre todo el poema.

En la página II, «Abîme», nombre casi propio con A mayúscula, indica un momento clave, una pauta en el texto al mismo tiempo que habla del (se refiere al) clinamen, estructura o forma del texto que sirve de meta-representación (Ricardou, 1987: 5) del fenómeno mismo de la escritura. Y nótese que en la misma estrofa (si se puede hablar en estos términos) aparecen otras dos palabras que tienen relación con el fenómeno antes descrito: «inclinaison» y «aile», de nuevo el contenido referido por el signo se funde con las estrategias performadas sobre el soporte. Gracias al clinamen se puede escribir y leer pasando de una página a otra, venciendo la barrera de división entre las páginas numeradas en el volumen; puede así más la disposición inclinada del texto (el clinamen de la escritura como efecto visual- 
pictórico) que el sentido normal y horizontalizado (occidentalizado, también) de la lectura. Se cumple también de este modo cierta pauta creativa del poeta, que se «conforma» con rellenar «tan sólo» un tercio de la página. Por ello el espacio en blanco adquiere un sentido inesperado, aunque calculado (y eso que siempre estuvo allí): ayudar a que el clinamen reproduzca lo más perfectamente posible el recorrido del ojo por el soporte, organizando los materiales depositados sobre su superficie. En la página derecha (461), «dresser le vol» (con dificultades...) viene a explicar la posición horizontal recobrada, una interpretación que sin duda puede ser completada por la valoración de una metáfora aérea y abismal, cuyos términos de representación mental encuentran sobre el soporte-página una organización de los signos configurada según formas que representarían una ala, el descenso, el horizonte, etc. En la página VIII se nos propone la visualización de cierta diseminación o dispersión de sus componentes. Dos clinamena ordenan el texto repartiendo desigualmente las masas de material escritural. Por cierto que esta descompensación de masas no tendría otra función que el poner en evidencia el cambio de unidad de soporte, es decir, que se anula la frontera de separación de las dos páginas de un solo plano lectural (téngase en cuenta que para el ojo ambas páginas tienen el mismo valor visual y que la lectura es una pauta añadida que cambia con las culturas). El primero de ellos, que comienza con «C'ETAIT» encima de «issu stellaire», es ya un mensaje casi cerrado y terminado que encuentra una aposición completa en un bloque a la derecha, donde se alternan las mayúsculas y el "bas de casse» casi con regularidad aritmética. No hay casi sensación de oblicuidad por la casi total tabularidad de la p. 473 y porque el texto de la izquierda se alinea junto a la costura divisoria como si se tratara de una glosa marginal o cita muy transitoria. En el segundo, destaca, en grandes mayúsculas, la cuarta parte del título diseminado, «LE HASARD», que convierte el imperfecto del primer clinamen en el condicional hipotético de "CE SERAIT». Aquí la oblicuidad es fácilmente consumible pues el clinamen ya tiene una disposición inclinada en la parte izquierda, con lo cual la presencia de «LE HASARD» atrae rápidamente el ojo y se transcurre así más suavemente hasta la parte final - en cursiva-, donde el tema de la pluma parece cerrar un proceso circular inaugurado anteriormente. En la página III, un clinamen de la parte izquierda deja vacilante el término «un» junto a la costura, permitiendo el juego combinatorio doble con «envahit» y «coule», o quizá pudiendo buscar también el sustantivo que se encuentra justo debajo y uniendo con otro clinamen de elevado lirismo y conformando un trayecto válido, aunque no único, que el lector puede recorrer a placer gracias a un punto de partida muy polisémico con el que es fácil viajar por el texto. A lo largo del texto se detectan algunos clinamena abreviados o aligerados, ya sea por su extensión o por el impacto visual que producen; hasta tal punto que no sería excesivo considerar como clinamen la presencia de «N'ABOLIRA», en solitario en la página 465 y arrimado al rincón inferior izquierdo. Como no termina la página precisamente, al dejar el rincón opuesto en blanco (por donde fluirá la página siguiente), «N’ABO- 
LIRA» pertenece más a la página 464 que a la 465 (donde sin embargo se inscribe), pues la fuerza lectural ejercida por la disposición del clinamen le obliga a esta nueva adscripción absolutamente lógica y funcional si trabajamos siempre con el criterio de la página IV y no tanto con la división tradicional que quisiera reducir el impulso expansivo inherente a la escritura. En la página $V$ desaparecen las páginas 466 y 467 como si se sumergieran en el abismo blanco del soporte. Se trata de la escritura más subrayadamente oblicua de cuantas constituyen este poema, una delación del clinamen por ese «COMME SI-COMME SI» que atraviesa el texto y la página al mismo tiempo que sugiere una hipótesis o propuesta. La cursiva del «bas de casse» incita/invita al consumo ávido de esa oblicuidad radicalizada hasta el extremo, cualquier dimensión tendente a la verticalidad queda así definitivamnte anulada y sólo hay entonces una posibilidad: leer en una dirección oblicua-horizontal, veloz, ansiosa por encontrar de nuevo el inicio del texto en el otro "COMME SI» final, para cerrar/abrir el círculo de esa abducción propuesta al lector sin condiciones. "COMME SI-COMME SI» es también la figura del eco, de la especularidad por antonomasia, una rima plena de un lado a otro de la página total, del abismo, del mundo, «como si» de una cosmogonía de palabras se tratara. Se produce entonces la instantaneidad típica del consumo visual, una simultaneidad provocada por el rápido desplazamiento del ojo de un extremo a otro y que casi ignora la lectura detenida de cada palabra del texto, al mismo tiempo que recibe el impacto de una imagen geométrica y arquitectural antes que la traducción de los significantes a los objetos pactados. El verso «RIENN'AURA EU LIEU - QUE LE LIEU» de la página IX constituye, además de un caso peculiar de diseminación, un intento de estructurar el texto de la página en su totalidad con un solo movimiento que atraviesa todo el espacio del plano. Con cierta gradación de sus tres partes además. De modo que la frase, nueva negación del azar, parece referirse al mismo trámite del poema (véase idéntico caso para el título), lanzado, como también lo fue el dado, a un destino indescifrable e infinito en el que sólo habría el texto y lo blanco, la escritura interactivada con su soporte. Otra frase, otro verso, en la página $\mathrm{X}$, «EXCEPTÉ-PEUT-ETRE-UNE CONSTELLATION», construye también un clinamen con las mismas características, aunque más disimulado, por su integración más intensa en el conjunto del texto. Tal como ocurre en el escalonamiento abismal del clinamen «veillant-donnant-roulant-brillant», donde la disposición formal obtiene la colaboración de una homogeneidad morfológica de los significantes. En la página VII, a la derecha (p. 471), «rire - que-SI» constituye un clinamen esquelético, gradual y cuidadosamente estructurado, mientras el resto de la parte derecha parece establecer una alineación vertical con la «inexistente» costura del volumen, aunque el recorrido de la lectura difumine un tanto su consumo vertical. De esta manera se propone un juego de doble sistema de lectura, pues la ignorancia o no de la costura - como signo que cuenta o no en la estructuración del texto de la nueva página- hace que se vacile un tanto a la hora de elegir la trayectoria lectural, lo cual no es algo limitativo, sino la expresión primera de la polisemia y la potenciación de la capacidad significante del texto. 


\section{LOS JUEGOS TIPOGRÁFICOS}

Cuando de la forma y materialidad del signo escrito se trata, no se puede obviar detalles que, normalmente, ni siquiera se citan en una edición crítica y mucho menos por supuesto son sabidos por el lector. Se trata de la tipografía. Como si fuera algo extraño al libro y al texto, parece que el trabajo correspondiente a la tipografía fuese una cuestión de imprenta solamente. El propio Mallarmé escogió el tipo Didot (Papp 1989: 194), con variaciones de cuerpo que van del 10 al 60 . Lo cual no es excesivamente llamativo para el lector de hoy, ya que el carácter Didot se parece mucho a una Times New Roman, tipo corriente y de uso frecuentísimo en los textos actuales. No así respecto al cuerpo pues, mientras que el cuerpo 10 es el más corriente, los cuerpos $24 \mathrm{y}$, sobre todo, 60 , no son muy utilizados, como no sea para títulos o portada. La clave tipográfica de «Un coup de Dés.... consiste pues en continuas alternancias redondilla/cursiva, minúscula/mayúscula, cuerpos 10/24/60 que, además, organizan trayectos de lectura que pueden ser autónomos, dando lugar a combinaciones poco usuales, como una especie de "sintaxis tipográfica» (Butor 1964: 149-151) o partitura musical (Michaud 1971: 201) y tal como el mismo autor había previsto y explicado en su prefacio al poema: regulación de la entonación, subrayar el valor semántico, etc. Pocos años después, la poesía visual utilizará estas pautas como referencia para la creación.

El título diseminado, por ejemplo, se unifica en un cuerpo 60 de mayúscula. De este modo, su inscripción imbricada cuidadosamente en cuatro lugares diferentes del texto no es algo problemático, sino la auténtica espina dorsal de ese organismo llamado "Un coup de Dés...», ya que asegura la presencia del título (no repetido) a lo largo del poema y su mezcla efectiva con los otros signos que constituyen el texto. Otra unificación, esta vez de cuerpo 24 en mayúsculas, se utiliza para organizar la frase tendida de un extremo a otro de la página IX: sólo la tipografía resaltada permite esta lectura. En la página I, la homogeneidad de la mayúscula, salvo la variación de cuerpo 60/24 debido a la presencia de «JAMAIS» (fragmento del título), subraya la apuesta por la infinitud del lanzamiento, del comienzo desde la nada, del surgir del texto desde la atemporalidad del soporte blanco y vacío. Frente a la utilización típica de la redondilla, la página $\mathrm{V}$ presenta todo su texto en cursiva (incluidas las mayúsculas de cuerpo 24), como ayudando en la tarea del clinamen. Inclinación de la escritura, del texto y de la letra que, además, se continúa en las páginas VI y VII en su totalidad, para frenar su alocada marcha, de repente y frente a la costura del volumen, en la mitad de la página VIII. Esta característica de velocidad y tendencia progresistatextual de la cursiva (unida al clinamen en muchos casos, además) se reúne con la expresión de la hipótesis, del azar, de la posibilidad incierta en el infinito que supone toda apuesta y se recoge perfectamente en expresiones de hipótesis (p. V: «COMME SI»), de condición (p. VII: «SI») y de posi- 
bilidad (p. VIII: «CE SERAIT»), así como en temas muy determinados (la cifra, el azar) o el (ab)uso del subjuntivo (p. VIII, entre la cifra y el azar).

\section{EL SOPORTE: LA PÁGINA}

En el acto de la lectura lo que importa es el signo, lo escrito, lo descifrable por el saber leer; no interesa el no-signo sobre el que se realiza la inscripción del trazo. Ese no-signo que es el soporte tiene sin embargo para Mallarmé una importancia vital, pues el leitmotiv de «Un coup de Dés...» consiste precisamente en manipular su funcionamiento y alterar las relaciones que mantiene con el signo inscrito. Las dos páginas que siempre tenemos ante nosotros cuando abrimos cualquier libro no son dos, sino una, porque lo que se ve es un objeto plano, blanco (con trazos inscritos) sobre el que nuestra mirada puede desplazarse, un espacio, uno, delimitable como unidad y analizable como tal (aunque hagamos referencia a veces a las dos páginas, numeradas eso sí, que es como se estructura el volumen). Pero lo que vemos y que podemos leer es una página, una unidad. Ya Mallarmé, en su prefacio al poema (1945a: 455) hablaba de esa «página» cuyo consumo simultáneo e instantáneo adquiere, además, el protagonismo de la composición, pues el verso ya no es aquí la unidad de referencia. Desde el momento en que se convierte en un sistema que ayuda en la tarea de la significación, la página se hace signo; un signo muy específico de $57 \times 38$ $\mathrm{cms}$. (en la edición original) y que obliga a una lectura diferente, no lineal, sino dispersa, no caótica, sino espacial, porque ya no hay un orden lectural único que vaya instituyendo el sentido, sino una lluvia de sentidos múltiples que se entrecruzan formando una red o tejido, un texto. El papel recoge la huella (Mallarmé 1945c: 369), pero en este caso no se trata solamente de un signo más, porque la escritura se asocia a los márgenes, a los espacios en blanco, se hace dispersa y expansiva, se interactiva totalmente con la sustancia albísima de la hoja de papel, de modo que ambos, trazo y papel, formarán un nuevo signo que se deja recorrer por la mirada. Inmediatamente después vendrá la lectura y el signo gráfico será consumido (sustituido por lo que representaba), pero quedará siempre el soporte con los trazos del opus nigrum.

\section{EL TÍTULO: DINÁMICA DE DISEMINACIÓN}

El título de Un coup de Dés jamais n'abolira le Hasard no es solamente un título. Ya desde la primera página de la obra, «UN COUP DE DES», primera fase o entrega al soporte del material del título, pone de 
relieve la fuerte interactividad con la hoja blanca: ha quedado vacío todo el espacio de la izquierda, apenas $4 \mathrm{cms}$. sirven para separar ese fragmento del título de la costura apenas perceptible o conocida, sólo destacan unos trazos de letra Didot mayúscula de cuerpo 60... El título, que no es el título, denuncia el vacío y el soporte no ya como potencialidad para un posible texto que allí podría inscribirse, sino como acto mismo que significa: aquí no hay otro texto que no sea el título que no sea ya este mismo soporte. Pero se trata de un título ficticio (Derrida 1972: 71), un «pars pro toto» de una secuencia textual extendida y diseminada a lo largo de toda la composición. En este sentido el poema titulado «Un coup de Dés jamais n'abolira le Hasard» podría muy bien reducirse a una frase negativa y negadora de lo infinito que es el mismo título, pero que también está en el poema entero, ya que forma parte de él en cuatro fases o inscripciones específicas y, además, adquiere una función real e importante para la escritura/lectura. Esta iteración discursiva de los topos incluidos en el título-poema constituye una constricción lectural que produce un impulso re-escritural, es decir, el lector tiene ante sí una estructura deconstruida y manipulable; y es obvio que la capacidad de re-escritura aumenta a medida que los componentes del texto (las cuatro piezas del puzzle-título imbricadas con todo el tejido textual) son más detectables (cuerpo 60) y que el lector puede intervenir más fácilmente con una consigna escritural a partir de esas mismas estructuras propuestas en el texto.

Caja de página:

$1 / 4$

UN COUP DE DES

$1 / 2$

$3 / 4$

JAMAIS

LE HASARD

N'ABOLIRA

Como bien puede verse, las diferentes fases del título no se inscriben en el mismo lugar de la página. Se trata pues de una combinatoria interesante respecto de la reflexión (inédita hasta entonces) sobre el soporte y su función en la composición textual. Como si se insistiera en obtener un título o algo que efectúe esa misma función, «UN COUP DE DES», en la p. 457, antes del comienzo del poema pero ya dentro del poema, tiene una página en blanco por delante y otra por detrás, aislando en cierto modo esta fase de la inscripción del título y permitiendo su consumo como función del título, pero sin perder la interacción con las otras fases ni quedarse fuera del andamiaje del texto. Este título que es un texto que se inscribe por cuatro veces en el texto, constituye por sí mismo toda una sintaxis ondulatoria, una composición aislada y musical (Butor 1964: 149-150) encofrada en un todo dinámico, una partitura de formas dispuestas e inscritas para 
funcionar con un criterio nuevo y plástico, más propio de un producto gráfico y figurativo (un cartel por ejemplo) que de una composición lírica. La novedad y el hito mallarmeanos son esto precisamente.

\section{LOS ESPACIOS EN BLANCO}

En ausencia del texto la página blanca importa mucho y provoca la significación obligada del soporte, es decir, el soporte también es signo. Si el signo gráfico escritural representa la palabra, la voz, el decir algo, el espacio en blanco es el silencio, el vacío, la ausencia. Estamos pues ante una dinámica lleno/vacío, presencia/ausencia, en la que por un lado el signo requiere previamente el vacío para llenar algo que no era, y por otro, una vez que el signo se ha inscrito, el vacío sigue presente de algún modo por medio de esos espacios en blanco entre signos gracias a los cuales es posible leer, pues de lo contrario tendríamos una hoja negra, atiborrada de signos apiñados, sobrepuestos, tachándose los unos a los otros; el espacio en blanco delimita el signo gráfico tanto o más que el trazo. Pero en «Un coup de Dés...» la dinámica del espacio en blanco alcanza niveles nunca vistos. No se trata ya solamente de las dos páginas vacías delante y detrás del título, sino de la vasta extensión ( $2 / 3$ del texto total) que llega a ocupar la ausencia de signos gráficos inscritos; lo cual además no es considerado como algo extraordinario, pues Mallarmé (1945a: 455) lo justifica totalmente en su prefacio: «je ne transgresse cette mesure, seulement je la disperse». La página 458 , justo detrás del fragmento del título que funciona como título, es una página en blanco, su texto es el no-texto que significa el soporte y la separación del título y el poema, que dice una pausa, ya que se trata de la no-escritura y que se ralentiza el consumo del texto interponiendo un fragmento de espacio (la página blanca) entre el título y el incipit del poema que, además, es otro fragmento del título. La presencia y funcionamiento de la página en blanco, antípoda de la página negra del Tristram Shandy de Sterne supone un grado de la escritura en el vacío, una apuesta contra las leyes vigentes de la representación de la lengua que, aquí, es un elemento más de un discurso multifuncional lingüístico, plástico, arquitectónico y muy cercano al tipo de representación operativo en pintura; su fusión simbiótica con la representación estándar constituye un gesto escritural revolucionario sin precedentes. Si la página escrita responde en esencia a un funcionamiento visual de lleno/vacío, Mallarmé desafiará las leyes vigentes del texto, de modo que en la página IV podamos encontrar una ruptura de la especularidad contrapuesta (línea frente a línea) típica de cualquier escrito: masas de texto muy estratificadas a la izquierda (la página 464 se parece excepcionalmente a una página normal) frente al desafío vacuo de la derecha, que presenta tan sólo el soporte con la inscripción de «N'ABOLIRA» en el rincón inferior izquierdo, como acabando el texto de 
la página anterior. Este mismo tipo de descompensación provocada por el efecto visual se repite en casi todas las páginas del poema, con mayor o menor impacto, y será especialmente significativo (y significante) en las páginas 468 (al forzar una oblicuidad protagonizada e impulsada por el clinamen) y 476 , donde un clinamen llega a vaciar la página, provocando la «huida» del texto hacia la derecha, pero sin la aceleración del caso anterior, pues sólo se pretende un vertido de grafos de una página a otra (estamos en un mismo plano espacial), para mostrar eficazmente la dinámica lleno/vacío y la capacidad significante del soporte.

El poema «Un coup de Dés jamais n'abolira le Hasard» no es un poema, es un verso único tendido de un extremo a otro de un espacio, de un volumen, la obra, el libro. Una «palabra absoluta» (Derrida 1974: 368379 ), en vez de estructuras reiterativas de versos superpuestos los unos sobre los otros para representar engañosamente el «éter transparente» (Derrida, ib.) de un mensaje que se quiere trascendente. La linealidad tradicional del mensaje lírico se transforma aquí en una contigüidad rigurosa y organizada, cuya pretensión diseminadora no es rebelarse contra un orden establecido, o no sólo esto, sino sobre todo mostrar una nueva relación compositiva y distribucional, como bien lo demuestra por ejemplo el título articulado con todo el texto. La tentativa de Mallarmé en este «coup de dés» va más allá (o quizá más acá) de la literatura y constituye todo un tratado sobre la escritura y su inscripción, sobre el signo y sus relaciones con el soporte. Cuando hablamos de que el soporte es un plano y que sobre él se inscriben los signos, es evidente que nos estamos refiriendo, en cierto sentido, a la técnica de los signos de la pintura. Que la escritura no ha seguido el funcionamiento habitual o convencional que suele tener en la página de un texto literario y se ha desviado hacia un comportamiento que tiene que ver más con una dimensión plástica o, lo que es lo mismo, que la escritura ha sacado a la luz todas las capacidades de su dimensión gráfica, donde la organización del material sígnico escritural supone una inscripción basada en las pautas siguientes: a) la unidad de soporte es aquí el plano de un volumen abierto, por tanto la división en páginas (varios planos contiguos sin relación formal entre sí) que funciona tradicionalmente en el libro es sustituida por una unidad diferente y de mayor operatividad; b) el depósito de la escritura sobre ese plano no se guía por una sintaxis típica ni por una disposición en líneas estándares, sino que utiliza la tipografia para compensar/descompensar la construcción del espacio producido en/sobre el soporte, organiza la frase o las secuencias sintagmáticas buscando ecos, resonancias, especularidades, significaciones atípicas, etc. Se escribe por tanto como se pinta. Los resultados son espectaculares y se obtiene un texto altamente significante en el que el lector-visualizador dispone de un aparato complejo (que no complicado) a efectos de la consecución de múltiples significaciones. Opera aperta hasta el extremo que no duda en permitir y provocar otras actuaciones además de la lectura. La significación, además de desgajarse de las palabras, viene inducida por efectos sensoriales (visua- 
les, táctiles) que tienen como referencia un signo materializado en una realidad física obviada convencionalmente por la escritura/lectura, pero que aquí es la protagonista de una dimensión plástica revolucionaria. Además, Mallarmé ha ensayado con éxito una especie de «poemización» de la dispositio, una sintaxis de la materialidad textual que utiliza los componentes escriturales para obtener una significancia (signifiance) de ese texto diseñado de forma distinta. La lectura será diferente entonces: además de leer de modo convencional, habrá que visualizar masas de texto relacionadas por una proximidad flagrantemente buscada, se perderá la linealidad lectural del texto y recorreremos el plano del volumen abierto con itinerarios inéditos y sorprendentes; lectura tabular, lúdica y polisémica, cuya suma significante total nos parece más rica y atractiva. Porque el texto aparece como deconstruido, mostrando su organización y funcionamiento y poniendo a nuestra disposición componentes que podrían ser utilizados como matrices para ulteriores actos de re-escritura. El texto ha descubierto así su faceta de entidad que puede travestirse en forma icónica, como el clinamen o trazo inclinado compuesto de material textual que no duda en representar, como una mancha sobre la hoja, la deriva típica de lo escrito.

\section{Referencias}

ButOR, M. (1964): «Le livre comme objet». En Essais sur le roman. París: Gallimard. CORTÁzAR, J. (1985): Salvo el crepúsculo. Madrid: Alfaguara.

DERrIDA, J. (1972): La dissémination. Paris: Seuil.

- (1974): «Mallarmé». En Tableau de la littérature française, vol. III. París: Gallimard.

KristeVA, J. (1974): La révolution du langage poétique. Paris: Seuil.

Mallarmé, S. (1945a): «Préface pour Un coup de Dés...». En Oeuvres Complètes. H. Mondor y G. Jean-Abry (eds.). París: Gallimard/Pléiade.

- (1945b): «Un coup de Dés jamais n’abolira le Hasard». Ib.

- (1945c): «Quant au livre». Ib.

MichaUd, G. (1971): Mallarmé. París: Hatier.

PAPP, T. (1989): «De la page mallarméenne à l'écran poétique». En Le texte et son inscription, R. Laufer (ed.). París: Eds. du C.N.R.S.

RICARDoU, J. (1987): «Eléments de Textique I». Conséquences $\mathbf{n}^{\circ} 10$. 